\title{
Severe mental illness and type 2 diabetes: using theory and research evidence to develop complex behaviour change interventions
}

This paper provides a critical review of the literature on severe mental illness (SMI) and type 2 diabetes and presents an argument for the development of theory-based interventions tailored specifically for people with these co-existing conditions. Individuals with SMI are at an increased risk of physical health problems, with a high prevalence of type 2 diabetes among this population. Yet it is estimated that up to $70 \%$ of cases of diabetes among people with SMI are undiagnosed (Holt \& Mitchell, 2015) which has many clinical implications for the individuals themselves and the healthcare professionals involved in their care. Changes to lifestyle can significantly reduce the risk of developing diabetes in the first place and also reduce the risks of complications from the condition. However, lifestyle changes relating to diet, exercise and smoking can be particularly challenging for people with SMI. There is good evidence that educating people with diabetes about the disease in general and behaviour change to manage the condition can have a positive impact on clinical outcomes in the general population (Steinbekk et $a l$, 2012). However much less is known regarding the effectiveness and appropriateness of existing diabetes self-management programmes for people with SMI. This essay will present an argument for the use of theory and research evidence to strengthen the development and implementation of new interventions specifically for people with SMI and type 2 diabetes.

\section{Severe mental illness}

Severe mental illness (SMI) describes a range of long term mental disorders such as schizophrenia, schizoaffective disorders, bipolar disorder and depression with psychotic features. It is well established that people with a SMI have poorer physical health (Leucht $e t$ al, 2007; De Hert et al, 2011a) and that multimorbidity (defined as the presence of two or more long-term conditions) is common, with the presence of a mental health disorder 
associated with a greater number of co-existing physical health conditions (Barnett et al, 2012). De Hert et al (2011a) have highlighted that people with SMI are susceptible to many different physical health problems and that this is further exacerbated by additional risk factors. These include: psychotropic medications (antipsychotics, antidepressants and mood stabilizers) that frequently lead to weight gain and metabolic alterations linked to the use of second-generation antipsychotics; individual lifestyle factors such as poor diet, a lack of exercise and high levels of smoking; and disparities in access and utilization of health care services.

Research conducted in different countries has demonstrated that individuals with SMI are likely to die at a younger age than the general population, predominantly as a result of their physical health conditions. A population based study by Lawrence et al (2013) examined the records from 292,585 psychiatric patients registered with mental health services in Western Australia over a 20 years period (1985-2005). The researchers found the life expectancy gap to be 16 years for men and 12 years for women compared to the general population, with $77.7 \%$ of these early deaths among people with mental health problems attributed to physical health conditions. A large case register study conducted in Denmark, Finland and Sweden (Nordentoft et al, 2013) followed a cohort of 270,770 psychiatric patients who had been admitted to psychiatric hospital during a six year period (2000-2006) and found that life expectancy among women with mental health problems was 15 years shorter than the general population in the three countries and among men, 20 years shorter. A smaller case register study with 31,719 people with SMI conducted in South London (Chang et al, 2011), found that the people with SMI died prematurely by up to 17.5 years compared to people without SMI. These studies demonstrate an international trend of excess mortality among people with mental health problems compared to the general population. 
It is widely established that individuals with SMI are a vulnerable population who face a number of socioeconomic and health inequalities ( Thornicroft, 2011; Shiers et al, 2015). There is a growing body of evidence that inequalities can be in part attributed to disparities in healthcare provision which explain much of the excess mortality among this population (De Hert et al 2011a). Laurence \& Kisely (2010) identified three main areas where barriers to effective healthcare provision exist: systemic-level issues; provider related issues; and individual factors. The systemic-level issues relate to organisational features of health systems that include the geographic, managerial and resource separation of mental health services from other physical healthcare services. As a result, the care pathways for people with a SMI and physical health problems are often complex and fragmented, with a lack of integration between different specialities and patients required to navigate different services across primary and secondary care, mental health and other specialist services for physical conditions. As highlighted by Wallace et al (2015), patients with complex multimorbidity often see many different health care professionals across multiple healthcare sites and as a consequence, communication between these various providers is frequently lacking which can have a negative impact on patient outcomes.

Despite the widespread knowledge that individuals with SMI are at an increased risk of a large number of physical health disorders, the screening and assessment of physical health at an international level remains poor (De Hert et al, 2011b; Hardy et al 2011; 2013). Stigma and discrimination also act as barriers to accessing healthcare for people with SMI, in terms of the responsiveness of health services and professionals (De Hert et al, 2011b). This also relates to the issue of diagnostic overshadowing, when physical health problems are reframed 
as symptoms of mental illness, which has been experienced by mental health service users during encounters with health professionals (Nash, 2014).

Barriers to receiving appropriate and timely healthcare provision are also attributed to the attitudes, training and confidence of health professionals (Lawrence \& Kisely, 2010). Several cross-sectional studies have investigated the physical health care knowledge and training of mental health nurses in the UK (Nash, 2005; Howard \& Gamble 2011; Robson et al 2013) and Australia (Happell et al 2013) and have identified varying levels of preparation, involvement and confidence. A literature review of nine studies that focused on the role of mental health nurses in providing physical health care for people with SMI identified limitations in support and training in this area together with uncertainties about role and responsibilities (Blythe \& White, 2012). Similar findings have been found among primary care professionals, who report a lack of knowledge to care effectively for people with SMI (Lester et al, 2005).

Finally regarding individual factors, it is well known that modifiable lifestyle factors such as smoking, poor diet, lack of exercise, substance and alcohol consumption are highly prevalent among people with SMI and are significant risk factors for excess morbidity and mortality (De Hert et al 2011a). It is also important to recognise that for people with a SMI their physical health may be a low priority, with the motivation to change their lifestyle or seek help a particular challenge, especially when mentally unwell (Buhagiar et al, 2011; Robson \& Gray, 2007). Illness related characteristics of SMI, such as cognitive impairment, social isolation and suspicion of health services and professionals are also recognised as barriers to the recognition and management of physical health problems (Hert et al, 2011b). 


\section{Diabetes mellitus and SMI}

Diabetes mellitus is a common long-term condition and its prevalence is increasing globally, affecting an estimated $8.8 \%$ of adults - 415 million people worldwide - and taking up $12 \%$ of international health expenditure (International Diabetes Federation, 2015). Diabetes is associated with severe complications; it is the leading cause of new cases of kidney disease, lower extremity amputations, and blindness not related to injury among adults (Lee et al, 2015; Molitch et al 2015). Type 2 diabetes is the most common type of diabetes accounting for around $90 \%$ of cases (Diabetes UK, 2016). This condition typically develops during adulthood, usually in people over the age of 40 years, but younger onset is becoming more common.

Diabetes is a metabolic disorder that is characterized by poorly regulated blood glucose levels, which may arise from defects in insulin secretion (insulin deficiency), or in its action (insulin resistance), or both. Raised blood glucose (hyperglycaemia) can initially be controlled by lifestyle changes to diet and exercise, but given the progressive nature of type 2 diabetes it is likely that most individuals will ultimately require pharmacological intervention as well. This may initially be with oral hypoglycaemic drugs to increase the production or uptake of insulin and, if the disease remains uncontrolled, insulin therapy (Diabetes UK, 2016). Multimorbidity is common among people with type 2 diabetes; a recent retrospective study of medical records of 1,389,016 patients in the USA with type 2 diabetes found that $97.5 \%$ of the patients had at least one comorbid condition in addition to type 2 diabetes and $88.5 \%$ had at least two. The most common conditions in these patients included: hypertension in $82.1 \%$; obesity in $78.2 \%$; hyperlipidemia in $77.2 \%$; chronic kidney disease in 24.1\%; and cardiovascular disease (CVD) in 21.6\% (Iglay et al, 2016). 
Existing research suggests a 2-3 fold increased incidence of type 2 diabetes among individuals with SMI ( Osborn et al, 2008; De Hert et al, 2011a) and a prevalence of 12\% for people receiving antipsychotic treatment (Holt \& Mitchell, 2015). Individuals with SMI are at increased risk of being diagnosed with type 2 diabetes for a number of reasons, including: the effects of anti-psychotic medications, which lead to weight gain for $80 \%$ of patients (Alvarez-Jimenez et al, 2008); lifestyle factors, such as poor diet, obesity and physical inactivity (Holt \& Mitchell, 2015; Stubbs et al, 2015); and high rates of smoking (Lawrence et al, 2009). The existence of significant health inequalities among this population is evident. For example, research conducted in East London (Mathur et al, 2012) found that those with SMI and diabetes were more likely to smoke, be obese and less likely to have had retinopathy screening than those without SMI. In addition, less than half of the population met the glycaemic control target of $\mathrm{HbA} 1 \mathrm{c}<7.5 \%$, a standardised measure of glycaemic control and directly associated with the risk of diabetic complications (Diabetes UK, 2016). Furthermore, among people diagnosed with diabetes, those with SMI have been found to have higher mortality (Vinogradova et al, 2010) and a greater risk of complications requiring specialist treatment (Becker \& Hux, 2011) compared to people without SMI.

The high prevalence of type 2 diabetes among individuals with SMI has many clinical implications, including: the importance of screening for diabetes in this population; implementing strategies to reduce the risk of developing diabetes; providing integrated care for those who develop diabetes; and developing effective interventions for the management of diabetes among people with SMI (Pendlebury \& Holt, 2010). As previously discussed, research suggests that up to $70 \%$ of cases of diabetes in people with SMI are undiagnosed (Holt \& Mitchell, 2015) thus there is a strong imperative for the screening of diabetes for people with SMI. Indeed this is recommended by a number of national and international 
bodies, including: the National Institute for Health and Care Excellence (NICE) in the UK (2014a; 2014b); the Joint European Societies, the American Diabetes Association, Canadian Diabetes Association and Australian Diabetes Association (Pendlebury \& Holt, 2010). However, while such guidelines are important in terms of setting standards for care, compliance is often low (Lawrence \& Kisely, 2010).

\section{Self-management education for people with SMI \& type 2 diabetes}

A crucial part of diabetes care is the requirement for an individual to change their lifestyle, particularly in terms of healthy eating, taking exercise and not smoking. The provision of education and training for people with diabetes about the disease and how to change their behaviour is considered pivotal in supporting them to manage their condition (Davies et al, 2008). This recommendation is reflected in guidance in different countries; In the UK and Canada, guidelines for type 2 diabetes ( National Institute for Health and Care Excellence, 2008; Canadian Diabetes Association, 2013) recommend that structured education is integrated into routine care and should be offered to all patients with diabetes. In the USA, the national standards for diabetes self-management education go further and specify that for people with SPMI (serious and persistent mental illness), the educational content should address the special issues involved with living with both a SPMI and diabetes (McDevitt, 2003).

Diabetes self-management education is defined as the process of facilitating the knowledge, skills and abilities necessary for diabetes self-care (Funnell et al, 2009). There is good evidence that diabetes self-management education can have a positive impact on clinical, lifestyle and psychosocial outcomes in the general population, at least in the short term (Deakin et al, 2005; Steinsbekk et al, 2012; Pal et al, 2013). However, much less is known 
about the effectiveness of self-management programmes for people with SMI and type 2 diabetes, as many existing studies have either excluded those with SMI or do not specify the inclusion criteria in relation to SMI. Furthermore, in those studies that do not exclude on the basis of mental illness, there tends to be no examination of whether a diagnosis of SMI has an impact on the effectiveness of the interventions.

The authors are aware of just one randomised controlled trial (RCT) conducted in the USA (McKibbin et al 2006), that purposely recruited adults over 40 years of age with schizophrenia and type 2 diabetes to test the feasibility and preliminary effectiveness of a diabetes self-management education programme designed for this population. The trial recruited 64 adults and compared usual care plus information leaflets with a 24 week education programme delivered once a week for 90 minutes called Diabetes Awareness and Rehabilitation Training (DART). DART covered basic diabetes education and information about lifestyle including healthy eating and exercise. A total of 57 patients completed the study, with nearly $40 \%$ of participants attending all sessions and $80 \%$ attendance at half of the intervention sessions. There were significant changes in BMI and bodyweight among the intervention group, with a mean weight loss of 5lb compared to the usual care group who gained an average of 6lb. The programme also led to reductions in waist circumference, diabetes knowledge, self-efficacy and activity levels immediately following the intervention. At six months follow up (12 months after the DART programme started) the DART participants were still significantly improved in terms of weight loss and BMI, waist circumference and diabetes knowledge compared to the usual treatment group (McKibbin et al 2010). However, the programme failed to improve HbA1c or report on whether there were any diabetes-related complications, increases in mortality, adverse events, or affected healthrelated quality of life or whether the programme was cost effective. 
The encouraging findings of this study (McKibbin et al 2006; 2010) suggest that people with a SMI can remain engaged in a group- based self-management programme over a number of weeks and there can be significant improvements in some important clinical outcomes, such as weight loss. These findings are supported by a systematic review of three studies of lifestyle interventions for individuals with schizophrenia or schizoaffective disorder and type 2 diabetes (Cimo et al, 2012). This review suggests that lifestyle interventions for this population can be effective in managing type 2 diabetes, particularly interventions that address diet and exercise. Although with only three studies in this review, it must be acknowledged that the evidence-base for such interventions for this population remains limited. Consequently, we still do not know which elements of these interventions are the most effective and for which patients. However, what is clear is that any intervention packages targeted at this population need to be tailored towards the unique challenges experienced by individuals with SMI. These include: loss of energy and weight gain associated with the use of antipsychotics; cognitive deficits which can make incorporating a daily routine for taking medication and eating regularly difficult; low motivation, particularly when acutely unwell; socioeconomic and health inequalities; and perceived or actual stigma and discrimination (De Hert et al, 2011b; Holt \& Mitchell, 2015).

\section{Developing complex behaviour change interventions: the need for theory}

In order to develop new and effective interventions for people with SMI and type 2 diabetes, utilizing the best available evidence and appropriate theory is essential. Theories are important tools in the social and natural sciences (May et al, 2009) and are equally important in health research. The Medical Research Council (MRC) framework for the development and evaluation of complex interventions (Medical Research Council, 2000) has been highly 
influential in this area of research by providing a theoretical guide to the development and implementation of new interventions (Craig et al, 2008). There is a growing body of evidence that suggests the explicit use of theory in the formulation of behaviour change interventions results in more effective interventions than those lacking a theoretical base (Glanz \& Bishop, 2010; Michie \& Prestwich, 2010). This is particularly relevant for complex behaviour change interventions, such as self-management programmes, that involve many interacting components relating to the individuals themselves, their long-term condition(s) and the systems (e.g. health systems and society) within which they are situated.

Theories define a core set of ideas that relate to explaining and making predictions about how things work (Imenda, 2014). The use of theory in the development of interventions to change behaviour can therefore provide a richer understanding of the target population, their characteristics and behaviour and the context and systems within which they are situated, that both enable and prevent behaviour change. Examples of theories that have been used to underpin diabetes self-management interventions include: the health belief model (Becker \& Janz, 1985); the transtheoretical model (stages of change) (Ruggiero, 2000); social cognitive theory (Bandura, 1986); the self-regulation model (Leventhal et al, 2003); and the social ecological model (Glanz \& Bishop, 2010).

Michie \& Prestwich (2010) promote the role of theory in the development of interventions and the potential benefits this can bring. Firstly, they propose that theory can help identify constructs (key concepts in the theory) which are considered to be causally related to a particular behaviour and are therefore appropriate targets for an intervention. They argue that by changing the constructs that cause the particular behaviour, this can lead to behaviour change. Secondly, Michie \& Prestwich (2010) suggest that when the collection of empirical 
data is guided by a theoretical framework, this facilitates the accumulation of evidence on effectiveness of a theory across different populations, behaviours and contexts. Thirdly, they argue that when interventions are underpinned by theory, and this is clearly documented, this provides a greater understanding of why interventions are effective or not. This is particularly important in terms of being able to identify the core intervention components, also known as the 'active ingredients,' that bring about behaviour change.

An extensive literature and guidance exists on the range of potential approaches to changing behaviour (National Institute for Health and Care Excellence, 2007; National Institute for Health and Care Excellence, 2014c) and different frameworks have been developed for designing and evaluating complex behaviour change interventions, with theory central to this process (Michie and Prestwich, 2010). The MRC framework for the development and evaluation of complex interventions is one that is widely used (Medical Research Council, 2000). This framework recommends a structured and systematic approach to the development, evaluation and implementation of complex interventions (see Figure 1) and stresses the importance of undertaking each stage thoroughly. The MRC guidance also recommends that interventions are developed with a good theoretical understanding of how the intervention is likely to bring about the desired change(s) in behaviour (Craig et al, 2008). However, a critique of the MRC and similar frameworks is that they do not provide guidance regarding which theories are most appropriate and how to use them (Michie and Prestwich, 2010).

\section{Insert Figure 1 here}


There are a vast number of behaviour change theories reported in the literature (see Hobbs $e t$ $a l, 2011)$ so it is immensely helpful for researchers that consolidated theoretical frameworks have been developed to synthesis broader domains and concepts across a range of theories. One such framework is the Theoretical Domains Framework (TDF), an integrative framework of theories of behaviour change that was developed to synthesise behaviour change theories and constructs in a format to make theory more accessible and usable for researchers working across different disciplines, countries and healthcare systems (Cane et al, 2015). The TDF (Cane et al, 2012) has synthesised 33 theories of behaviour change down to 14 theoretical domains that have been found to influence behaviour. These domains are: knowledge; skills; beliefs about capabilities; beliefs about consequences; emotions; environmental context and resources; social influences; memory, attention and decision processes; social/professional role and identity; reinforcement; intentions; goals; optimism; behavioural regulation. By using the TDF to understand which of the 14 domains act as barriers and enablers to performing a behaviour, we can then identify the behaviour change techniques (BCTs), using established mapping techniques (Cane et al, 2015), that can be targeted within an intervention in order to change behaviour.

\section{Using theory to develop a complex behaviour change intervention for people with SMI} and type 2 diabetes

We are currently using the Theoretical Domains Framework (TDF) as a theoretical framework for two linked research studies that are exploring the barriers and enablers which inhibit and/or support individuals with SMI to manage their diabetes. These studies constitute part of the development phase of a structured programme of research, following the MRC framework (Medical Research Council, 2000) as a theoretical guide to develop an 
effective self-management education and support intervention for people with SMI and type 2 diabetes.

A qualitative interview study with people with SMI and type 2 diabetes, carers, and healthcare professionals (mental health nurses, psychiatrists, GPs, practice nurses, GPs, diabetologists and diabetes specialist nurses) aims to identify the experiences of these different stakeholder groups regarding the management of type 2 diabetes among people with SMI. The interview topic guide for these semi-structured interviews is based on the TDF (Cane et al, 2012), with additional questions designed to allow participants to discuss topics relevant to their experience of diabetes and mental illness. The interview data will be analysed using the 14 domains of the TDF; this process will allow us to identify the key behaviours people with diabetes and SMI find most challenging. It will also identify the specific beliefs and attitudes which service users and healthcare professionals hold which may impact upon effective diabetes self-management and the theoretical constructs and models which determine these behaviours. Furthermore, conducting qualitative research as part of the development phase of a behaviour change intervention introduces an important 'person-based approach' that Yardley et al (2015) suggest provides a valuable in-depth understanding of the psychosocial context of users and their views on the behavioural elements of the proposed intervention. Such an approach, the researchers argue, is a systematic way of addressing the user experience of intended behaviour change techniques and can enhance the use of theory and evidence-based approaches to intervention development.

The second study is a cross-sectional survey of people with SMI and type 2 diabetes and healthcare professionals (mental health nurses, psychiatrists, GPs, practice nurses, GPs, 
diabetologists and diabetes specialist nurses) who provide care for this population. The findings from the interviews will be mapped onto theories of behaviour change and the constructs within these theories will support the development of the questionnaire for this survey. It is anticipated that the survey will identify the psychological constructs that predict, in the case of service users, the challenging aspects of diabetes self-management such as selfmonitoring of blood glucose, adherence to a healthy diet, exercise, adherence to medication, titration of insulin (if relevant), foot care and attendance for regular screening including diabetic retinopathy and general check-ups. From the healthcare professionals it is anticipated that the survey will provide a better understanding of the current practices being implemented for this population and an understanding of the psychological constructs that predict these practices in the delivery of diabetes care.

Alongside these two studies we are undertaking a Cochrane review on the effectiveness of diabetes self-management interventions for people with Type 2 diabetes (McBain et al, 2014). This will extend the scope of the previous review undertaken by Cimo and colleagues (2012) to include people with personality disorder, bipolar disorder and depression with psychotic features. In addition to looking at the evidence for their effectiveness, the review will also provide us with the opportunity to describe, using established reporting systems, the active components of these interventions and the theoretical frameworks within which they were developed (Michie \& Prestwich, 2010; Michie et al, 2013).

It is important for our research to be informed by the views and participation of people with lived experience of both conditions (SMI and diabetes). Since the inception of the research, from the initial generation of research ideas, design and conduct of the two studies discussed, we have been working collaboratively with an established group of mental health service 
users and carers called SUGAR (Simpson et al, 2014), members of the Diabetes Research Network as well as service users and family members/carers with experience of both SMI and type 2 diabetes. This way of collaborative working is not only good research practice (Gradinger et al, 2013) but also helps to ensure that the interventions that are developed and implemented are feasible, acceptable and relevant to the service users, carers and public involved.

\section{Conclusion: the role of theory in mental health interventions}

In this paper we have outlined the current research evidence in the fields of SMI and type 2 diabetes and identified some of the many challenges around the management of type 2 diabetes for people with SMI and the healthcare professionals who are involved in their care. Some significant clinical implications have been identified, particularly the need for screening for diabetes among people with mental health problems and the ongoing monitoring and support from healthcare professionals. It is clear that the challenges of addressing multimorbidity for healthcare professionals are complex, with healthcare systems and clinical guidelines usually structured around a single disease focus that frequently fails to consider the person holistically (Wallace et al, 2015). For mental health nurses, previous research has shown that they frequently lack the adequate knowledge and skills to provide integrated mental and physical health care and that training and support for mental health nurses in physical healthcare is required (Blythe \& Whyte, 2012; Robson et al, 2013).

To effectively support and empower people with SMI to manage their diabetes, as well as the mental health nurses and other healthcare professionals who support them, we consider that evidence-based and theoretically informed interventions need to be developed to meet the health needs of this population. In this paper we have presented an argument for the use of 
theory and research evidence to underpin the development and implementation of a diabetes self-management education and support programme for people with SMI. We have also highlighted the importance of a clearly documented and structured approach to future research, using a theoretically driven framework such as the MRC framework for the development and evaluation of complex interventions (Craig et al, 2008). The evidence from this growing research field strongly suggests that a theory-driven and evidence-based approach will strengthen the development and implementation of effective, acceptable and relevant interventions for people with SMI and type 2 diabetes.

\section{References}

Alvarez-Jimenez, M., Hetrick, S.E., González-Blanch, C., Gleeson, J.F. \& McGorry, P.D. (2008) Non-pharmacological management of antipsychotic-induced weight gain: systematic review and meta-analysis of randomised controlled trials. British Journal of Psychiatry, 192(8), 101-107.

Bandura, A. (1986) Social Foundations of Thought and Action: A Social Cognitive Theory. Prentice-Hall, Englewood Cliffs, N.J.

Barnett, K., Mercer, S.W., Norbury, M., Watt, G., Wyke, S. \& Guthrie, B. (2012) Epidemiology of multimorbidity and implications for health care, research, and medical education: A cross-sectional study. The Lancet, 380 (9836), 37-43.

Becker, M. H \& Janz, N.K. (1985) The Health Belief Model Applied to Understanding Diabetes Regimen Compliance. The Diabetes Educator, 11 (1), 41-47 
Becker, T. \& Hux, J. (2011) Risk of acute complications of diabetes among people with schizophrenia in Ontario, Canada. Diabetes Care, 34, 398-402.

Blythe, J. \& White, J. (2012) Role of the mental health nurse towards physical health care in serious mental illness: an integrative review of 10 years of UK literature. International Journal of Mental Health Nursing, 21, 193-201.

Buhagiar, K., Parsonage, L., \& Osborn, D. P. (2011) Physical health behaviours and health locus of control in people with schizophrenia-spectrum disorder and bipolar disorder: a crosssectional comparative study with people with non-psychotic mental illness. BMC Psychiatry, $11,104$.

Canadian Diabetes Association (2013) Clinical Practice Guidelines for the Prevention and Management of Diabetes Mangement in Canada. Canadian Journal of Diabetes, 37, A3A13.

Cane, J., O'Connor, D., \& Michie, S. (2012) Validation of the theoretical domains framework for use in behaviour change and implementation research. Implementation Science, 7, (1) 37.

Cane, J., Richardson, M., Johnston, M., Ladha, R, \& Michie, S. (2015) From lists of behaviour change techniques (BCTs) to structured hierarchies: Comparison of two methods of developing a hierarchy of BCTs. British Journal of Health Psychology, 20, 130-150.

Chang, C. K., Hayes, R. D., Perera, G., Broadbent, M. T., Fernandes, A. C., Lee, W. E. et al. (2011) Life expectancy at birth for people with serious mental illness and other major disorders from a secondary mental health care case register in London. PLoS.One., 6, e19590. 
Cimo, A., Stergiopoulos, E., Cheng, C., Bonato, S., \& Dewa, C. S. (2012) Effective lifestyle interventions to improve type II diabetes self-management for those with schizophrenia or schizoaffective disorder: A systematic review. BMC Psychiatry, 12, 24.

Craig, P., Dieppe, P., Macintyre, S., Michie, S., Nazareth, I., \& Petticrew, M. (2008) Developing and evaluating complex interventions: the new Medical Research Council guidance. BMJ, 337, a1655.

Davies, M. J., Heller, S., Skinner, T. C., Campbell, M. J., Carey, M. E. \& Cradock, S. (2008) Effectiveness of the diabetes education and self management for ongoing and newly diagnosed (DESMOND) programme for people with newly diagnosed type 2 diabetes: cluster randomised controlled trial. British Medical Journal, 336(7642), 491-5.

De Hert, M., Correll, C.U., Bobes, J., Cetkovich-Bakmas, M., Cohen, D., Asai, I. et al. (2011a) Physical illness in patients with severe mental disorders. I. Prevalence, impact of medications and disparities in health care. World Psychiatry, 10, 52-77.

De Hert, M., Cohen, D., Bobes, J., Cetkovich-Bakmas, M., Leucht, S., Ndetei, D.M. et al. (2011b) Physical illness in patients with severe mental disorders. II. Barriers to care, monitoring and treatment guidelines, plus recommendations at the system and individual level. World Psychiatry, 10, 138-151.

Deakin, T., McShane, C. E., Cade, J. E., \& Williams, R. D. (2005) Group based training for selfmanagement strategies in people with type 2 diabetes mellitus. The Cochrane Database of Systematic Reviews, 2. 
Diabetes UK. (2016) What is type 2 diabetes. Available at: https://www.diabetes.org.uk/Guide-todiabetes/What-is-diabetes/What-is-Type-2-Diabetes/ (Accessed: $11^{\text {th }}$ April 2016).

Funnell, M.M., Brown, T.L., Childs, B.P., Haas, L.B., Hosey, G.M. Jensen, B. et al. (2009) National Standards for Diabetes Self-Management Education. Diabetes Care, 32, (Supplement 1), s87-s94.

Glanz, K. \& Bishop, D. B. (2010) The Role of Behavioral Science Theory in Development and Implementation of Public Health Interventions. Annual Review of Public Health, 31, 399418.

Gradinger, F., Britten, N., Wyatt, K., Froggatt, K., Gibson, A., Jacoby, A., Lobban, F., Rawcliffe, T. \& Popay, J. (2013) Health Expectations, 18, 661-675

Happell, B., Scott, D., Nankivell, J. \& Platania-Phung, C. (2013) Screening physical health? Yes! But...: nurses' views on physical health screening in mental health care. Journal of Clinical Nursing, 22, 2286-2297.

Hardy, S, Hinks, P. \& Gray, R. (2013) Screening for cardiovascular risk in patients with severe mental illness in primary care: a comparison with patients with diabetes. Journal of Mental Health, 22 (1), 42-50.

Hardy, S., White, J., Deane, K. \& Gray, R. (2011) Educating healthcare professionals to act on the physical health needs of people with serious mental illness: a systematic search for evidence. Journal of Psychiatric and Mental Health Nursing, 18, 721-727. 
Hobbs, L., Campbell, R., Hildon, Z. \& Michie, S. (2011) Behaviour change theories across Psychology, Sociology, Anthropology and Economics: A systematic review. Psychology and Health, 26 (Suppl. 1), 31

Holt, R.I. \& Mitchell, A.J. (2015) Diabetes mellitus and severe mental illness: mechanisms and clinical implications. Nature Reviews Endocrinology, 11(2), 79-89.

Howard, L. \& Gamble, C. (2011) Supporting mental health nurses to address the physical health needs of people with serious mental illness in acute inpatient care settings. Journal of Psychiatric and Mental Health Nursing, 18 (2), 105-112.

Iglay, K., Hannachi, H., Howie, P.J., Xu, J., Li, X., Engel, S.S. et al. (2016) Prevalence and Coprevalence of Comorbidities Among Patients With Type 2 Diabetes Mellitus. Current Medical Research Opinion, 17(3), 1-44. [Epub ahead of print]

Imenda, S. (2014) Is There a Conceptual Difference between Theoretical and Conceptual Frameworks? Journal of Social Science, 38(2), 185-195.

International Diabetes Federation (2015) [Online] IDF Diabetes Atlas, 7th edition. Available at: http://www.diabetesatlas.org/ (Accessed: 14th April 2016)

Lawrence, D. \& Kisely, S. (2010) Inequalities in healthcare provision for people with severe mental illness. Journal of Psychopharmacology, 24(Supplement 4), 61-68. 
Lawrence, D., Hancock, K.J. \& Kisely, S. (2013) The gap in life expectancy from preventable physical illness in psychiatric patients in Western Australia: retrospective analysis of population based registers. British Medical Journal, 346, f2539.

Lawrence, D., Mitrou, F., \& Zubrick, S. R. (2009) Smoking and mental illness: results from population surveys in Australia and the United States. BMC Public Health, 9, 285.

Lee, R., Wong, T.Y. \& Sabanayagam, C. (2015) Epidemiology of diabetic retinopathy, diabetic macular edema and related vision loss. Eye and Vision, 2, 17.

Lester, H., Tritter, J. Q., \& Sorohan, H. (2005) Patients' and health professionals' views on primary care for people with serious mental illness: focus group study. British Medical Journal, $330,1122$.

Leventhal, H., Brissette, I. \& Leventhal, E.A. (2003) The common-sense model of self-regulation of health and illness. In: Cameron, L.D., Leventhal, H. (Eds.) The Self-Regulation of Health and Illness Behaviour. Routledge, London

Leucht, S., Burkard, T., Henderson, J., Maj, M. \& Sartorius, N. (2007) Physical Illness and Schizophrenia: A Review of the Evidence. Acta Psychiatrica Scandinavica, 116, 317-333.

Mathur, R., Hull, S. A., Boomla, K., \& Robson, J. (2012) Ethnic differences in primary care management of diabetes and cardiovascular disease in people with serious mental illness. British Journal of General Practice, 62, e582-e588. 
May, C.R., Mair, F., Finch, T., MacFarlane, A., Dowrick, C., Treweeket, S. et al. (2009)

Development of a theory of implementation and integration: normalization process theory. Implementation Science, 4, 29.

Medical Research Council (2000) A Framework for the Development and Evaluation of RCTs for Complex Interventions to Improve Health. MRC, London.

McBain, H., Mulligan, K., Haddad, M., Flood, C., Jones, J., \& Simpson, A. (2014) Selfmanagement interventions for type 2 diabetes in adult people with severe mental illness (Protocol). The Cochrane Database of Systematic Reviews (11)

McDevitt, J. (ed.) (2003) Diabetes Management in the Context of Serious and Persistent Mental Illness. In: Clinical practice recommendations evidence-based guidelines for integrated care, Expert Reviewed Edition. Chicago: The Nursing Institute, College of Nursing University of Illinois.

McKibbin, C.L., Golshan, S., Griver, K., Kitchen, K. \& Wykes, T.L. (2010) A healthy lifestyle intervention for middle-aged and older schizophrenia patients with diabetes mellitus: A 6month follow-up analysis. Schizophrenia Research, 121, 203-206.

McKibbin, C.L., Patterson, T.L., Norman, G., Patrick, K., Jin, H., Roesch, S. et al. (2006) A lifestyle intervention for older schizophrenia patients with diabetes mellitus: a randomized controlled trial. Schizophrenia Research, 86, 36-44.

Michie, S. \& Prestwich, A. (2010) Are interventions theory-based? Development of a theory coding scheme. Health Psychology, 29, (1), 1-8. 
Michie, S., Richardson, M., Johnston, M., Abraham, C., Francis, J., Hardeman, W. et al. (2013) The behavior change technique taxonomy (v1) of 93 hierarchically clustered techniques: building an international consensus for the reporting of behavior change interventions. Annals of Behavioral Medicine, 46, 81-95.

Molitch, E., Adler, A.I., Flyvberg, A., Nelson, R. G., So, W-Y., Wanner, C. et al (2015) Diabetic kidney disease: A clinical update from Kidney Disease: Improving Global Outcomes. Kidney International, 87, (1), 20-30.

Nash, M. (2005) Physical care skills: a training needs analysis of inpatient and community mental health nurses. Mental Health Practice, 9, 20-23.

Nash, M. (2014) Mental health service users' experiences of diabetes care by Mental Health Nurses: an exploratory study. Journal of Psychiatric Mental Health Nursing, 21(8), 715-23.

National Institute for Health and Care Excellence. (2007) Behaviour change: the principles for effective interventions. [Online] London, NICE. Available from: http://www.nice.org.uk/nicemedia/live/11868/37987/37987.pdf. (Accessed: $18^{\text {th }}$ November 2015).

National Institute for Health and Clinical Excellence. (2008) The Management of Type 2 diabetes (update) (Clinical guideline 66). [Online] London, NICE. Available from: https://www.nice.org.uk/guidance/cg66. (Accessed: $18^{\text {th }}$ November 2015).

National Institute for Health and Care Excellence. (2014a) Psychosis and Schizophrenia in Adults: Prevention and management [Online] London, NICE. Available from: https://www.nice.org.uk/guidance/cg178 (Accessed: 14 ${ }^{\text {th }}$ April 2016). 
National Institute for Health and Care Excellence. (2014b) Bipolar Disorder: Assessment and Management [Online] London, NICE. Available from:

https://www.nice.org.uk/guidance/cg185 (Accessed: 14 ${ }^{\text {th }}$ April 2016).

National Institute for Health and Care Excellence. (2014c) Behaviour Change: Individual Approaches. [Online] London. NICE. Available from: http://www.nice.org.uk/Guidance/PH49. (Accessed: $18^{\text {th }}$ November 2015).

Nordentoft, M., Wahlbeck, K., Hällgren, J., Westman, J., Ösby U, Alinaghizadeh, H. et al. (2013) Excess Mortality, Causes of Death and Life Expectancy in 270,770 Patients with Recent Onset of Mental Disorders in Denmark, Finland and Sweden. PLoS ONE, 8, 1: e55176.

Osborn, D. P., Wright, C. A., Levy, G., King, M. B., Deo, R., \& Nazareth, I. (2008) Relative risk of diabetes, dyslipidaemia, hypertension and the metabolic syndrome in people with severe mental illnesses: systematic review and metaanalysis. BMC Psychiatry, 8, 84.

Pal, K., Eastwood, S. V., Michie, S., Farmer, A. J., Barnard, M. L., Peacock, R. et al. (2013) Computer-based diabetes self-management interventions for adults with type 2 diabetes mellitus. Cochrane Database of Systematic Reviews.

Pendlebury, J. \& Holt, R.I. ( 2010) Managing diabetes in people with severe mental illness. Journal of Diabetes Nursing, 92010 (14), 328-338.

Robson, D. \& Gray, R. (2007) Serious mental illness and physical health problems: a discussion paper. International Journal of Nursing Studies, 44, 457-466.

Robson, D., Haddad, M., Gray, R., \& Gournay, K. (2013) Mental health nursing and physical health care: A cross-sectional study of nurses' attitudes, practice, and perceived training needs 
for the physical health care of people with severe mental illness. International Journal of Mental Health Nursing, 22(5), 409-417.

Ruggiero, L. (2000) Helping people with diabetes change behavior: From theory to practice. Diabetes Spectrum, 13(3), 125.

Shiers, D., Bradshaw, T. \& Campion, J. (2015) Health inequalities and psychosis: time for action. British Journal of Psychiatry, 207 (6), 471-473

Simpson, A., Jones, J., Barlow, S. and Cox, L. \& Service User and Carer Group Advising on Research (SUGAR) (2014) Adding SUGAR: Service user and carer collaboration in mental health nursing research. Journal of Psychosocial Nursing and Mental Health Services, 52, (1), 22-30.

Steinsbekk, A., Rygg, L., Lisulo, M., Rise, M. B., \& Fretheim, A. (2012) Group based diabetes self-management education compared to routine treatment for people with type 2 diabetes mellitus. A systematic review with meta-analysis. BMC Health Services Research, 12, 213.

Stubbs, B., Vancampfort, D., De Hert, M. \& Mitchell, A.J. (2015) The prevalence and predictors of type two diabetes mellitus in people with schizophrenia: a systematic review and comparative meta-analysis. Acta Psychiatrica Scandinavica, 132, 144-157.

Thornicroft, G. (2011) Physical health disparities and mental illness: the scandal of premature mortality. British Journal of Psychiatry, 199, 441-442.

Vinogradova, Y., Coupland, C., Hippisley-Cox, J., Whyte, S., \& Penny, C. (2010) Effects of severe mental illness on survival of people with diabetes. British Journal of Psychiatry, 197 (4), 272-277. 
Wallace, E., Salisbury, C., Guthrie, B., Lewis, C., Fahey, T. \& Smith, S.M. (2015) Managing patients with multimorbidity in primary care. British Medical Journal, 350, 176.

Yardley, L., Morrison, L., Bradbury, K. \& Muller, I. (2015) The person-based approach to intervention development: Application to digital health-related behaviour change interventions. Journal of Medical Internet Research, 17 (1), e30 